\title{
Comparing effects of polyunsaturated fatty acids derived from marine and plant sources on endothelial cell inflammation
}

\author{
Ella Baker, Elizabeth Miles and Philip Calder \\ University of Southampton, Southampton, United Kingdom
}

\section{Abstract}

High consumption of $\omega-3$ long chain polyunsaturated fatty acids (PUFAs) has long-term health benefits. The principal dietary source of these $\omega-3$ PUFAs (eicosapentaenoic acid, EPA and docosapentaenoic acid, DHA) is seafood, particularly oily fish. However current fish stocks are decreasing, indicating a need for alternative sources of bioactive PUFAs. Plant-derived $\omega-3$ PUFAs (alphalinolenic acid, ALA and stearidonic acid, SDA) may be able to provide land-based sustainable sources, but their functionality has been underexplored.

Anti-inflammatory effects of ALA and SDA were compared to EPA and DHA in cultured EA.hy926 endothelial cells. Cells were treated with PUFAs $(10,25$ and $50 \mu \mathrm{M})$ for 48 hours prior to stimulation with tumour necrosis factor for 24 hours. PUFAs incorporation was measured by gas chromatography and inflammatory responses were measured by ELISA, RT-PCR, western blot and flow cytometry. Adhesion of THP-1 monocytes to EA.hy926 cells was determined using a static adhesion assay.

All PUFAs were incorporated into EA.hy926 cells in a dose-dependant manner $(10$ and $50 \mu \mathrm{M})$. Pre-treatment with ALA, SDA, EPA and DHA $(50 \mu \mathrm{M})$ had differential effects on inflammatory responses in EA.hy926 cells depending on PUFA and response examined.

EA.hy926 cells pre-treated with SDA had lower concentrations of soluble ICAM-1 $(p<0.05)$; however EPA and DHA resulted in greater reduction $(p<0.0001)$. EPA and DHA pre-treated EA.hy926 cells had significantly lower concentrations of IL-6 $(p<0.0001)$, IL-8 $(\mathrm{p}<0.0001)$ and MCP-1 $(\mathrm{p}<0.05, \mathrm{p}<0.01)$. ALA pre-treatment did not significantly affect any of the cytokines examined. Lower cell surface expression of ICAM-1 $(\mathrm{p}<0.05)$, was seen for EA.hy926 cells pre-treated with SDA again to a lesser extent than EPA and DHA $(p<0.001, p<0.0001)$, with no effect seen after ALA treatment.

EA.hy926 cells pre-treated with ALA had significantly higher relative gene expression of NFKB $(\mathrm{p}<0.05)$, as well as a tendency for more phosphorylated NFKBp65 protein $(\mathrm{p}<0.06)$. In contrast, EA.hy926 cells pre-treated with DHA had significantly less phosphorylated NFKB $(\mathrm{p}<0.0001)$. EA.hy926 cells with DHA treatment had significantly higher relative gene expression of PPARa $(p<0.05)$. SDA and EPA had no effect on expression of either of the genes or proteins examined.

Finally pre-treatment with ALA, SDA and DHA all resulted in reduced adhesion of THP-1 monocytes to EA.hy926, however this effect not observed with EPA.

Marine derived PUFA, particularly DHA, resulted in potent anti-inflammatory effects within this endothelial cell model. Of the two plant derived PUFAs, SDA treatment lead to some anti-inflammatory effects, which were not seen after treatment with ALA.

\section{Conflict of Interest}

There is no conflict of interest 